\title{
Binomial Transforms of the k-Lucas Sequences and its Properties
}

Pooja Bhadouria

School of Studies in Mathematics, Vikram University, Ujjain, India

Pooja.kajal@yahoo.co.in
Deepika Jhala*

School of Studies in Mathematics, Vikram University, Ujjain, India

Jhala.deepika@gmail.com

*corresponding author
Bijendra Singh

School of Studies in Mathematics, Vikram University, Ujjain, India

bijendrasingh@yahoo.com

\section{Article history: \\ Received May 2013 \\ Accepted June 2013 \\ Available online June 2013}

\begin{abstract}
In this paper, we employ several class of transforms like binomial, k- binomial and rising transforms to the k- Lucas sequence. Moreover, we investigate some interesting properties between the so-obtained new sequences and the k-Lucas sequence.
\end{abstract}

Mathematics Subject classification: 11B39, $11 \mathrm{~B} 37$.

Keywords: k-Fibonacci sequence, k-Lucas sequence, binomial, k-binomial, and rising transforms.

\section{Introduction}

Fibonacci and Lucas numbers have long interested mathematicians for their intrinsic theory and applications. For rich applications of these numbers in science and nature one can see the citations in [9, $11,12]$. For instance the ratio of two consecutive of these numbers converges to the golden section $\alpha=\frac{1+\sqrt{5}}{2}$. The application of the golden ratio appears in many research areas particularly in physics, engineering, architecture, nature, art and computer science etc.

The well known Fibonacci $\left\{F_{n}\right\}$ and Lucas $\left\{L_{n}\right\}$ sequences are defined by:

$$
F_{n}=F_{n-1}+F_{n-2} \text { and } L_{n}=L_{n-1}+L_{n-2},
$$


where $F_{1}=F_{2}=1$ and $L_{1}=2, L_{2}=1$ respectively.

Fibonacci sequence has been generalized in many ways $[4,5]$. Here, we use the following one-parameter generalization of the sequence.

For any positive integer number $k \geq 1$, the $k^{\text {th }}$ Fibonacci sequence say $\left\{F_{k, n}\right\}$ is defined recurrently by

$F_{k, 0}=0, F_{k, 1}=1$ and $F_{k, n+1}=k F_{k, n}+F_{k, n-1}$ for $n \geq 1$

In [6] Falcon introduced k-Lucas sequence generated by k-Fibonacci sequence whose recurrence relation is given by

$L_{k, 0}=2, L_{k, 1}=k$ and $L_{k, n+1}=k L_{k, n}+L_{k, n-1}$ for $n \geq 1$

In [4], these general k- Fibonacci numbers were found by studying the recursive application of two geometrical transformations used in the well known four-triangle longest-edge (4TLE) partition. In [8], authors apply several transforms to the k-Fibonacci sequences and deduce some properties between them. Also, in [3] authors establish some new properties of k-Fibonacci and k-Lucas numbers in terms of binomial sums. Falcon and Plaza studied 3-dimensional k-Fibonacci spirals with a geometric point of view in [7]. Some identities for the k-Lucas numbers may be found in [6].

The focus of this paper is to apply binomial transforms and its generalization (like k-binomial transform and rising transforms) to the k-Lucas sequence and evaluate several properties between k-Lucas sequence and obtained new sequences.

In [2], the binomial transform $\mathrm{B}$ of the integer sequence $T=\left\{a_{0}, a_{1}, a_{2}, a_{3}, \ldots\right\}$, which is denoted by $B(T)=\left\{b_{n}\right\}$ and defined by:

$$
b_{n}=\sum_{i=0}^{n}\left(\begin{array}{l}
n \\
i
\end{array}\right) a_{i}
$$

The first k-Lucas sequence defined in (1.2) indexed in the On-Line Encyclopedia of Integer sequence (OEIS) [10] are:

$$
\begin{aligned}
& \left\{L_{1, n}\right\}=\{2,1,3,4,7, \ldots\} \\
& \left\{L_{2, n}\right\}=\{2,2,6,14,24, \ldots\} \\
& \left\{L_{3, n}\right\}=\{2,3,11,36,119, \ldots\} \\
& \left\{L_{5, n}\right\}=\{2,638,234,1442, \ldots\} \\
& \left\{L_{4, n}\right\}=\{2,5,27,140,727, \ldots\}
\end{aligned}
$$

Now in [5], Pascal 2-triangle is presented and studied by means of the k-Fibonacci sequences, which is an extension of classical Pascal triangle for the Fibonacci sequence. Here we create the Pascal 2-triangle for the k-Lucas sequence. 
Figure 1. Pascal 2-triangle for the k-Lucas sequence

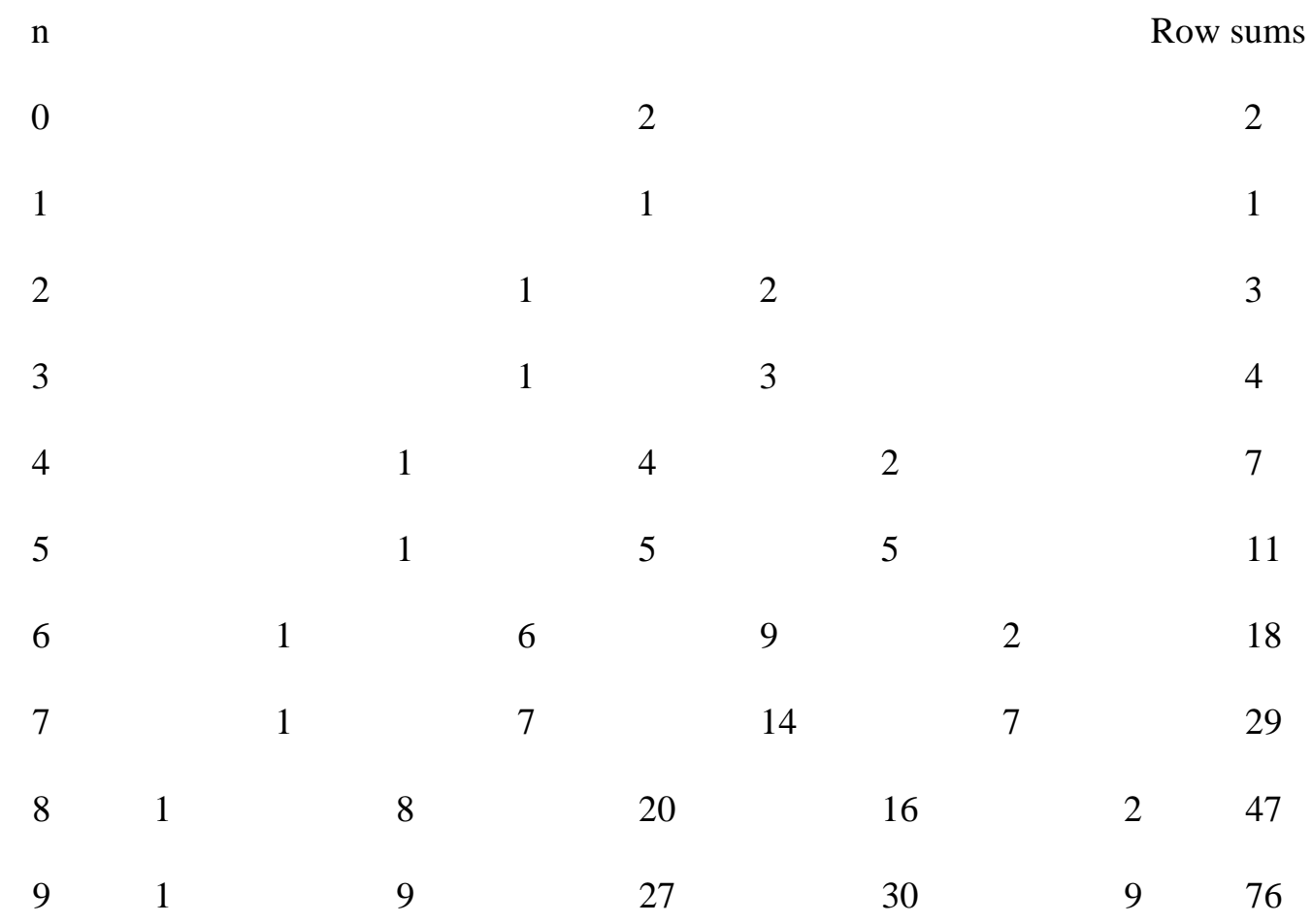

It is noted that the coefficients arising in the previous table are such a way that every side of the triangle is double, and for this reason this triangle will be called Pascal 2-triangle.

\section{Binomial Transform of the k-Lucas Sequences}

The binomial transform of the k-Lucas sequence $\left\{L_{k, n}\right\}$ is denoted by $B_{k}=\left\{b_{k, n}\right\}$ where

$$
b_{k, n}=\sum_{i=0}^{n}\left(\begin{array}{l}
n \\
i
\end{array}\right) L_{k, i}
$$

From the above definition the binomial transformation of the Lucas sequence $L_{n}=\{2,1,3,4,7,11, \ldots$.$\} is$ the sequence $B_{1}=\left\{b_{1, n}\right\}=\{2,3,7,18,47,123,322, \ldots .$.$\} , called bisection of Lucas sequence and also$ indexed as A005248 in OEIS.

The Binomial transform of the k-Lucas sequence are as follows-

$$
\begin{aligned}
& B_{1}=\{2,3,7,18,47, \ldots .\} \text { A } 005248 \\
& B_{2}=\{2,4,12,40,136, \ldots . .\} \text { A } 056236
\end{aligned}
$$




$$
\begin{aligned}
& B_{3}=\{2,5,19,80,343, \ldots .\} \\
& B_{4}=\{2,6,28,144,752, \ldots .\}
\end{aligned}
$$

Binomial transform of k-Lucas sequences can be obtained as application of the following theorem.

2.1 Theorem 1: Binomial transform of the $k$-Lucas sequence $B_{k}=\left\{b_{k, n}\right\}$ verifies the recurrence relation

$$
b_{k, n+1}=(k+2) b_{k, n}-k b_{k, n-1} \text { for } n \geq 1 \text {, }
$$

with initial conditions $b_{k, 0}=2$ and $b_{k, 1}=k+2$.

Proof: In the proof of this theorem we will use following lemma:

Lemma: The binomial transform of the $k$-Lucas sequence verifies the relation

$$
b_{k, n+1}=\sum_{i=0}^{n}\left(\begin{array}{l}
n \\
i
\end{array}\right)\left(L_{k, i+1}+L_{K, i}\right)
$$

From the above lemma and from equation (1.2), we have

$$
\begin{aligned}
b_{k, n+1} & =\sum_{i=1}^{n}\left(\begin{array}{l}
n \\
i
\end{array}\right)\left(k L_{k, i}+L_{K, i}+L_{k, i-1}\right)+\left(L_{k, 1}+L_{k, 0}\right) \\
& =\sum_{i=1}^{n}\left(\begin{array}{l}
n \\
i
\end{array}\right)\left(k L_{k, i}+L_{K, i}+L_{k, i-1}\right)+(k+2) \\
& =(k+1) \sum_{i=1}^{n}\left(\begin{array}{l}
n \\
i
\end{array}\right) L_{k, i}+\sum_{i=1}^{n}\left(\begin{array}{l}
n \\
i
\end{array}\right) L_{k, i-1}+k+2
\end{aligned}
$$

and therefore from equation (2.1), we can write

$$
b_{k, n+1}=(k+1) b_{k, n}+\sum_{i=1}^{n}\left(\begin{array}{l}
n \\
i
\end{array}\right) L_{k, i-1}-k
$$

Taking into account that $\left(\begin{array}{c}n-1 \\ n\end{array}\right)=0$, we have

$$
\begin{aligned}
b_{k, n} & =(k+1) b_{k, n-1}+\sum_{i=1}^{n-1}\left(\begin{array}{c}
n-1 \\
i
\end{array}\right) L_{k, i-1}-k \\
& =k b_{k, n-1}+\sum_{i=0}^{n-1}\left(\begin{array}{c}
n-1 \\
i
\end{array}\right) L_{k, i}+\sum_{i=1}^{n-1}\left(\begin{array}{c}
n-1 \\
i
\end{array}\right) L_{k, i-1}-k
\end{aligned}
$$




$$
\begin{aligned}
& =k b_{k, n-1}+\sum_{i=1}^{n}\left[\left(\begin{array}{c}
n-1 \\
i-1
\end{array}\right)+\left(\begin{array}{c}
n-1 \\
i
\end{array}\right)\right] L_{k, i-1}-k \\
& =k b_{k, n-1}+\sum_{i=1}^{n}\left(\begin{array}{l}
n \\
i
\end{array}\right) L_{k, i-1}-k
\end{aligned}
$$

Hence,

$$
\sum_{i=1}^{n}\left(\begin{array}{l}
n \\
i
\end{array}\right) L_{k, i-1}-k=b_{k, n}-k b_{k, n-1}
$$

Thus substitute this value in equation (2.4), we obtained the formula (2.2) that is

$$
b_{k, n+1}=(k+2) b_{k, n}-k b_{k, n-1} \text { for } n \geq 1 \text {. }
$$

\subsection{Binet's formula for the binomial transform of the k-Lucas sequence}

Binet's formula are well known in the Fibonacci number theory [1, 11]. Also, the Binet's formula for the k-Lucas numbers given in [6]. In our case, Binet's formula allows us to express the binomial transform of $\mathrm{k}$-Lucas sequence in the function of the roots $\sigma_{1}$ and $\sigma_{2}$ of the following characteristic equation associated to the recurrence relation (2.2), $\sigma^{2}-(k+2) \sigma+k=0$.

Proposition 2: The $\mathrm{n}^{\text {th }} \mathrm{k}$-Lucas number of binomial transform is given by $b_{k, n}=\sigma_{1}^{n}+\sigma_{2}^{n}$, where $\sigma_{1}$ and $\sigma_{2}$ are the roots of the equation (2.6), and $\sigma_{1} \succ \sigma_{2}$.

Proof: The roots of the characteristic equation (2.6) are:

$$
\sigma_{1}=\frac{k+2+\sqrt{k^{2}+4}}{2} \text { and } \sigma_{2}=\frac{k+2-\sqrt{k^{2}+4}}{2}
$$

The roots $\sigma_{1}$ and $\sigma_{2}$ verifies the relation such as $\sigma_{1} \cdot \sigma_{2}=k, \sigma_{1}+\sigma_{2}=k+2, \sigma_{1}-\sigma_{2}=\sqrt{k^{2}+4}$, and $\sigma_{1}=\alpha+1$ with $\alpha=\frac{k+\sqrt{k^{2}+4}}{2}$. Therefore the general term of the binomial transform of the k-Lucas sequence may be expressed in the form $b_{k, n}=C_{1} \sigma_{1}^{n}+C_{2} \sigma_{2}^{n}$ for some coefficients $C_{1}$ and $C_{2}$. Giving to $\mathrm{n}$ the values $n=0$ and $n=1$ it is obtained $C_{1}=C_{2}=1$, and therefore $b_{k, n}=\sigma_{1}^{n}+\sigma_{2}^{n}$.

\subsection{Generating function for the binomial transform of the k-Lucas sequence}

In this section, the generating function for the binomial transform of the k-Lucas sequence is given.

Let the binomial transform of the k-Lucas numbers are the coefficient of a potential series centered at the origin and consider the corresponding analytic function $b_{k}(x)$ such that 


$$
b_{k}(x)=b_{k, 0}+b_{k, 1} x+b_{k, 2} x^{2}+\ldots
$$

Then

$$
\begin{aligned}
& (k+2) x b_{k}(x)=(k+2) b_{k, 0} x+(k+2) b_{k, 1} x^{2}+(k+2) b_{k, 2} x^{3}+\ldots, \\
& k x^{2} b_{k}(x)=k b_{k, 0} x^{2}+k b_{k, 1} x^{3}+k b_{k, 2} x^{4}+\ldots
\end{aligned}
$$

Since from equation (2.2), $b_{k, n}=(k+2) b_{k, n-1}-k b_{k, n-2}, b_{k, 0}=2$, and $b_{k, 1}=k+2$, we obtain

$$
\left(1-(k+2) x+k x^{2}\right) b_{k}(x)=2-(k+2) x,
$$

and hence the generating function for the binomial transform of the k-Lucas sequence $\left\{b_{k, n}\right\}_{n=0}^{\infty}$ is

$$
b_{k}(x)=\frac{2-(k+2) x}{1-(k+2) x+k x^{2}}
$$

\subsection{Triangle of the binomial transform of the $k$-Lucas sequence}

In this section, we create an infinite triangle of numbers $S_{k}$ for each $k$ by using the following rule:

(i) The left diagonal of the triangle consists of the elements of the k-Lucas sequence, and

(ii) Any number off the left diagonal is the sum of the number to its left and the number diagonally above it to the left.

Then the sequence on the right diagonal is the binomial transform of the k-Lucas sequence.

For example, following figure shows the triangle $S_{4}$ for the 4-Lucas sequence and its binomial transform: 
We note that the sequence on the right diagonal $\{2,6,28,144,752, \ldots\}$ is the binomial transform of the 4-Lucas sequence, that is $B_{4}=\left\{b_{4, n}\right\}$, which was defined in the previous section. Also, every antidiagonal sequence $\left\{c_{k, n}\right\}$ of this triangle verify the recurrence relation, which is same as the k-Lucas sequence.

That is $\quad c_{k, n+1}=k c_{k, n}+c_{k, n-1}$.

From the above figure, we can say that all diagonal sequences satisfy the relation

$$
c_{4, n+1}=6 c_{4, n}-4 c_{4, n-1},
$$

and the antidiagonal sequences satisfy the relation

$$
c_{4, n+1}=4 c_{4, n}+c_{4, n-1}
$$

In [2], author consider the variations of the binomial transform and gives theorems and formulae related on it. In a similar manner, we now consider following variations of the binomial transform and apply it to the k-Lucas sequence.

\section{The k-binomial Transform of the k-Lucas Sequence}

The k-binomial transform $\mathrm{T}$ of the sequence $\left\{L_{k, n}\right\}$ is the sequence $T_{k}=\left\{t_{k, n}\right\}$, where $t_{k, n}$ is defined by:

$$
t_{k, n}=\left\{\begin{array}{cc}
\sum_{i=0}^{n}\left(\begin{array}{c}
n \\
i
\end{array}\right) k^{n} L_{k, i} & \text { for } k \neq 0 \text { or } n \neq 0 \\
0 & \text { if } k=0 \text { and } n=0
\end{array}\right\}
$$

From the above definition, we can write the first k-binomial transform of k-Lucas sequence, which are as follows:

$$
\begin{aligned}
& T_{1}=\{2,3,7,18,47, \ldots\}, \\
& T_{2}=\{2,8,48,320,2176, \ldots\}, \\
& T_{3}=\{2,15,171,2160,27783, \ldots\}, \\
& T_{4}=\{2,24,448,9216,192512, \ldots\},
\end{aligned}
$$

Here $T_{1}$ is 1-binomial transform coincides with the binomial transform $\mathrm{B}_{1}$.

3.1 Theorem 3: The $k$-binomial transform of the $k$-Lucas sequence verifies the recurrence relation $t_{k, n+1}=k(\mathrm{k}+2) \mathrm{t}_{k, n}-k^{3} t_{k, n-1} ; n \geq 1$, with initial conditions $t_{k, 0}=2$, and $t_{k, 1}=k(\mathrm{k}+2)$.

Proof: from the equation (2.1) and equation (3.1), we can write

$$
t_{k, n}=k^{n} b_{k, n}
$$


Now consider,

$$
\begin{aligned}
& t_{k, n+1}=k^{n+1} b_{k, n+1} \\
& t_{k, n+1}=k^{n+1}\left((\mathrm{k}+2) \mathrm{b}_{k, n}-k b_{k, n-1}\right)
\end{aligned}
$$

After solving this, we get

$$
t_{k, n+1}=k(\mathrm{k}+2) \mathrm{t}_{k, n}-k^{3} t_{k, n-1} \text {, for } n \geq 1 \text {. }
$$

\subsection{Generating function and Binet's formula for the k-binomial transform of the k-Lucas sequence Generating function:}

Similarly to that for the binomial transform (2.8), we can find the generating function for the k-binomial transform, which is

$$
t_{k}(\mathrm{x})=\frac{2-k(k+2) \mathrm{x}}{1-k(\mathrm{k}+2) \mathrm{x}+\mathrm{k}^{3} x^{2}} .
$$

\section{Binet's formula:}

Binet formula for the k-binomial transform of the k-Lucas sequence is given by

$t_{k, n}=\sigma_{1}^{n}+\sigma_{2}^{n}$, where $\sigma_{1}=\frac{k+2+\sqrt{k^{2}+4}}{2} k$ and $\sigma_{2}=\frac{k+2-\sqrt{k^{2}+4}}{2} k$. (The proof is same as that of binomial transform of k-Lucas sequence, which is in equation (2.7))

Here the roots $\sigma_{1}$ and $\sigma_{2}$ verify the relations:

$\sigma_{1}-\sigma_{2}=\sqrt{k^{2}+4}$ and $\sigma_{1}=k(\alpha+1)$, where $\alpha=\frac{k+\sqrt{k^{2}+4}}{2}$.

\subsection{Triangle for the k-binomial transform of the k-Lucas sequence}

In this section, create an infinite triangle of numbers in such a way that,

(i) The left diagonal consists of the k-Lucas numbers, and

(ii) Any number off the left diagonal is k-times the sum of the numbers to its left and diagonally above it to the left.

Then the right diagonal is the k-binomial transform $T_{k}$ of $\left\{L_{k, n}\right\}$.

For example: the following figure shows the triangle for the 4-Lucas sequence and its k-binomial transform:

2

$2 \quad 4 \quad 24$ 
3

4

5
76

322
18

376

1592
88

1856

7872
448

9216

38912192512.

Here the right diagonal sequence $\{2,24,448,9216,192512, \ldots\}$ is the k-binomial transform of the 4Lucas sequence, as in the above definition.

\section{The Rising k-binomial Transform of the k-Lucas Sequence}

The rising k-binomial transform $\mathfrak{R}$ of the k-Lucas sequence $\left\{L_{k, n}\right\}$ is the sequence $\mathfrak{R}_{k}=\left\{r_{k, n}\right\}$, where $\left\{r_{k, n}\right\}$ is defined by

$$
r_{k, n}=\left\{\begin{array}{cc}
\sum_{i=0}^{n}\left(\begin{array}{l}
n \\
i
\end{array}\right) k^{i} L_{k, i} & \text { for } k \neq 0 \\
0 & \text { if } k=0
\end{array}\right\}
$$

From the definition (4.1), we can write the first rising k-binomial transform of the k-Lucas sequence, which are as follows:

$$
\begin{aligned}
& \mathfrak{R}_{1}=\{2,3,7,18,47, \ldots\}, \\
& \mathfrak{R}_{2}=\{2,6,34,198,1154, \ldots\}, \\
& \mathfrak{R}_{3}=\{2,11,119,1298,14159, \ldots\}, \\
& \mathfrak{R}_{4}=\{2,18,322,5778,103682, \ldots\} .
\end{aligned}
$$

\subsection{Theorem 4: The rising k-binomial transform of the k-Lucas sequence satisfies the following} recurrence relation

$$
r_{k, n+1}=\left(\mathrm{k}^{2}+2\right) \mathrm{r}_{k, n}-r_{k, n-1}, \text { forn } \geq 1
$$

With initial conditions $r_{k, 0}=2, r_{k, 1}=k^{2}+2$.

Proof: In the proof of this theorem, we will use following proposition:

Proposition: If $\mathfrak{R}_{k}$ is the rising k-binomial transform of the k-Lucas sequence $\left\{L_{k, n}\right\}$, then $r_{k, n}=L_{k, 2 n}$ forn $\in N$, where $\left\{L_{k, 2 n}\right\}$ is called the bisection of the k-Lucas sequence.

Proof of the proposition: As we know that the Binet's formula for the k-Lucas sequence is

$L_{k, n}=\alpha^{n}+\beta^{n}$, where $\alpha=\frac{k+\sqrt{k^{2}+4}}{2}$, and $\beta=\frac{k-\sqrt{k^{2}+4}}{2}$. [6] 
Then $\quad r_{k, n}=\sum_{i=0}^{n}\left(\begin{array}{c}n \\ i\end{array}\right) k^{i} L_{k, i}=\sum_{i=0}^{n}\left(\begin{array}{c}n \\ i\end{array}\right) k^{i}\left(\alpha^{i}+\beta^{i}\right)=\sum_{i=0}^{n}\left(\begin{array}{c}n \\ i\end{array}\right)(\mathrm{k} \alpha)^{i}+\left(\begin{array}{c}n \\ i\end{array}\right)(\mathrm{k} \beta)^{i}$

$$
=(k \alpha+1)^{n}+(k \beta+1)^{n}=\alpha^{2 n}+\beta^{2 n}=L_{k, 2 n} \text {. (Since } k \alpha+1=\alpha^{2} \text { ) }
$$

Proof of the main theorem: From the definition (1.2), and the above formula (4.3), we get

$$
\begin{aligned}
& r_{k, n+1}=L_{k, 2 n+2}=k L_{k, 2 n+1}+L_{k, 2 n} \\
& =\left(\mathrm{k}^{2}+1\right) \mathrm{L}_{k, 2 n}+L_{k, 2 n}-L_{k, 2 n-2}=\left(\mathrm{k}^{2}+2\right) \mathrm{L}_{k, 2 n}-L_{k, 2 n-2}
\end{aligned}
$$

Therefore $r_{k, n+1}=\left(\mathrm{k}^{2}+2\right) \mathrm{r}_{k, n}-r_{k, n-1 .} \quad$ [from equation (4.3)]

From the equation (4.2), we can find out the Binet formula and the generating function for the rising kbinomial transform of the k-Lucas sequence, which is proved by similar method as in section 2 .

\subsection{Generating function and Binet's formula for the rising k-binomial transform of the k-Lucas sequence}

\section{Binet's formula:}

Binet formula for the rising $\mathrm{k}$-binomial transform of the k-Lucas sequence is given by

$$
r_{k, n}=\left(\sigma_{1}^{n}+\sigma_{2}^{n}\right)
$$

where $\sigma_{1}=\frac{k^{2}+2+\sqrt{k^{4}+4 k^{2}}}{2}=k \alpha+1$, and $\sigma_{2}=\frac{k^{2}+2-\sqrt{k^{4}+4 k^{2}}}{2}$.

\section{Generating function:}

The generating function for the rising k-binomial transform is given by

$$
r_{k}(\mathrm{x})=\frac{2-\left(k^{2}+2\right) \mathrm{x}}{1-\left(\mathrm{k}^{2}+2\right) \mathrm{x}+x^{2}}
$$

\subsection{Triangle for the rising k-binomial transform of the $k$-Lucas sequence}

In this section, we can create an infinite triangle of numbers in such a way that,

(i) The left diagonal consists of the k-Lucas numbers, and

(ii) Any number off the left diagonal is the sum of the number diagonally above it to the left and $\mathrm{k}$-times the number to its left.

Then the right diagonal is the rising k-binomial transform $\mathfrak{R}_{k}$ of the k-Lucas sequence $\left\{L_{k, n}\right\}$.

Here, we create the triangle for the 4-Lucas sequence and its rising k-binomial transform. 


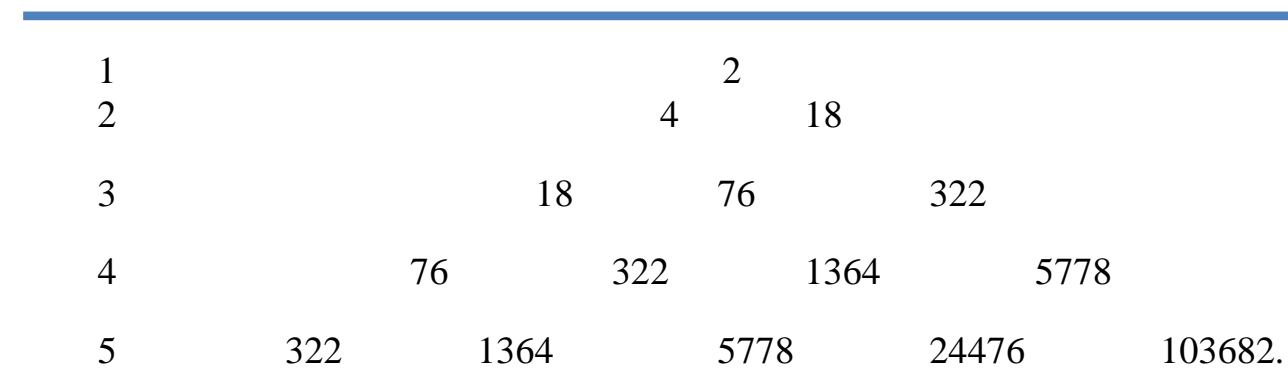

The right diagonal of this triangle $\{2,18,322,5778,103682, \ldots\}$ is the rising 4-binomial transform of the $\mathrm{k}$-Lucas sequence $\left\{\mathrm{L}_{k, n}\right\}$.

\section{Conclusion}

In this paper, we have established some known properties like Binet's formula, generating function and recurrence relation for the sequences related to several class of transforms like binomial, k-binomial and rising k-binomial transforms of the k-Lucas sequence.

\section{Acknowledgments}

The authors wish to express their gratitude to the referee for several helpful comments and suggestions concerning the paper.

\section{References}

[1] A. Stakhov, B. Robin, Theory of Binet formulas for Fibonacci and Lucas p-numbers, Chaos, Solitons \& Fractals, 27 (5), 1162-77, (2006).

[2] M.Z. Spivey \&L.L. Steil , The k-Binomial Transform and the Hankel Transform, Journal of Integer Sequences, Vol.9, (2006).

[3] N. Yilmaz, N. Taskara, K. Uslu \& Y. Yazlik, On The Binomial Sums of k-Fibonacci and k-Lucas Sequences, Numerical Analysis and Applied Mathematics, 341-344, (2011).

[4] S. Falcon, \& A. Plaza, On the Fibonacci k-numbers, Chaos, Solitons \& Fractals, 32(5), 1615-24, (2007).

[5] S. Falcon, \& A. Plaza, The Fibonacci Sequence and the Pascal 2-Triangle, Chaos, Solitons \& Fractals, 33 (1), 38-49, (2007).

[6] S. Falcon, On the k-Lucas Numbers, Int. J. Contemp. Math. Sciences, 2011, 6(21), 1039-1050.

[7] S. Falcon, \& A. Plaza, On the 3-Dimensional k-Fibonacci Spirals, Chaos, Solitons \& Fractals, 38(4), 993-1003, (2008).

[8] S. Falcon, \& A. Plaza, Binomial Transforms of the k-Fibonacci Sequence, International Journal of Nonlinear and Numerical Simulation, 10 (11-12), 1527-1538, (2009).

[9] S. Vajda, Fibonacci and Lucas Numbers and the Golden Section, Theory and Applications, John Wiley and Sons, New York, (1989). 
P. Bhadouria, D. Jhala, B. Singh / J. Math. Computer Sci. 8 (2014), 81 - 92

[10] Slone NJA, The Online Encyclopedia of Integer Sequences, 2006, www.research.att.com/ njas/sequences/.

[11] T. Koshy, Fibonacci and Lucas Numbers with Applications, John Wiley \& Sons, Inc., New York, (2001).

[12] V. E. Hoggat, Fibonacci and Lucas Numbers, Palo Alto, CA: Houghton, (1969). 